Supporting Information

\title{
Amplifiable Symmetry Breaking in Aggregates of Vibrating Helical Molecules
}

Fang Wang, Fuwei Gan, Chengshuo Shen, and Huibin Qiu*

School of Chemistry and Chemical Engineering, Frontiers Science Center for Transformative Molecules, State Key Laboratory of Metal Matrix Composites, Shanghai Jiao Tong University, Shanghai 200240, China.

E-mail: hbqiu@situ.edu.cn (H. Qiu). 


\section{Experimental section}

\subsection{Instruments and methods}

$C D$ spectra of suspension and solution samples were measured in quartz cuvettes (light path length $10 \mathrm{~mm}$ ) on a JASCO J-815 CD spectrometer. Temperature interval experiments were carried out with a temperature gradient of $2{ }^{\circ} \mathrm{C} / \mathrm{min}$ and waiting time of $60 \mathrm{~s}$ in the heating process. For the CD spectra of freeze-dried aggregates, the samples were first mixed with $\mathrm{KBr}$ and casted into plates, and then the plates were placed perpendicular to the light path of $C D$ spectrometer and rotated within the plate plane to rule out the possibility of birefringency and eliminate the possible angle dependence of the CD signal.

Circularly polarized luminescence (CPL) spectra were recorded in quartz cuvettes with an optical path length of $10 \mathrm{~mm}$ using a JASCO CPL-300 spectrometer.

UV-visible (UV-vis) absorption spectra were recorded using Shimadzu UV-2600 Spectrometer at $20^{\circ} \mathrm{C}$ in a $1 \mathrm{~mm}$ quartz cell.

The fluorescence spectra were recorded on a LS 55 from Perkin Elmer, Inc., USA.

SEM was performed on a JEOL JSM-7800F microscope with an accelerating voltage of $5 \mathrm{kV}$. Samples were prepared by dropping ca. $50-100 \mu \mathrm{L}$ of suspension onto a polished silicon wafer, followed by drying and coating with a thin layer of $A u$ to enhance the contrast.

Transmission electron microscopy (TEM) was performed on a JEM-1400 plus microscope at an accelerating voltage of $120 \mathrm{kV}$. The TEM samples were prepared by casting a small amount of sample onto a copper omentum and dried overnight in the air.

Single crystal $X$-ray diffraction measurements were performed on a Bruker SMART Apex II CCD-based X-ray diffractometer with Mo-Karadiation $(\lambda=0.71073 \AA)$. Powder Xray diffraction (PXRD) data were collected on a D8 Advance instrument from Bruker-AXS Company over $2 \theta$ range of $3.0^{\circ}-30.0^{\circ}$ at room temperature. Freeze-dried samples were cast on glass substrates for PXRD measurements.

\subsection{Materials}

All starting materials and solvents were of AR grade quality, which were purchased from commercial sources and used without any further purification. 2-Aza[4]helicene, 4aza[4]helicene, and naphtho[2,1-f]quinoline were synthesized according to a previous report and NMR data was in well agreement with the literature. ${ }^{\mathrm{S} 1}$ 


\section{Additional experimental data and figures}
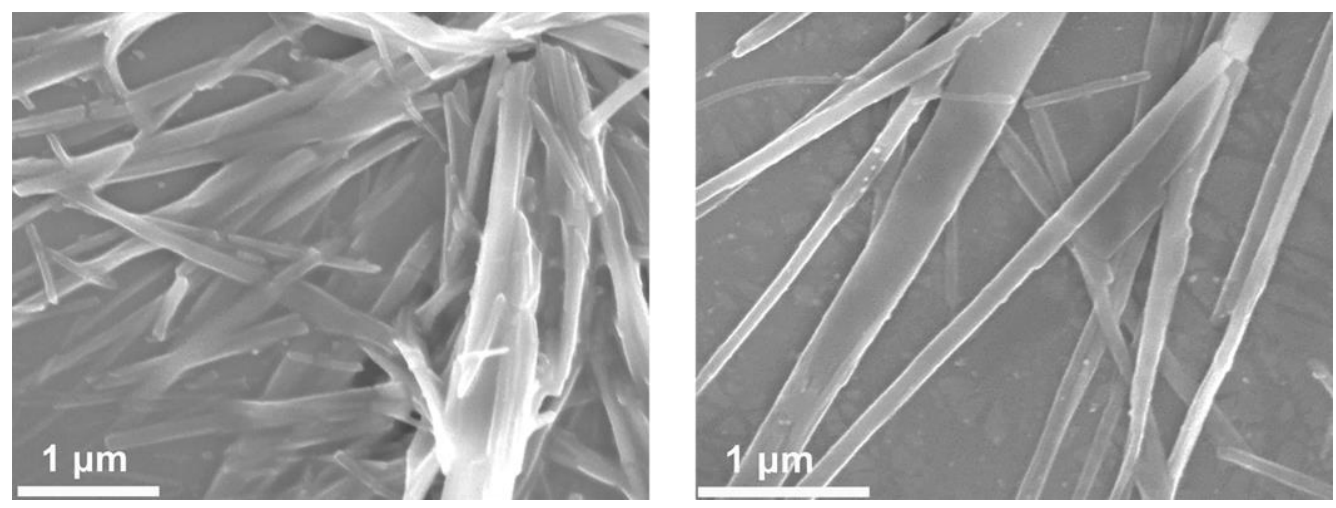

Figure S1. Additional SEM images of dried 2-A[4] $\mathrm{H}$ aggregates obtained in $\mathrm{DMF} / \mathrm{H}_{2} \mathrm{O}(1 / 99$, v/v) with a concentration of $1.3 \times 10^{-3} \mathrm{~mol} \cdot \mathrm{L}^{-1}$.
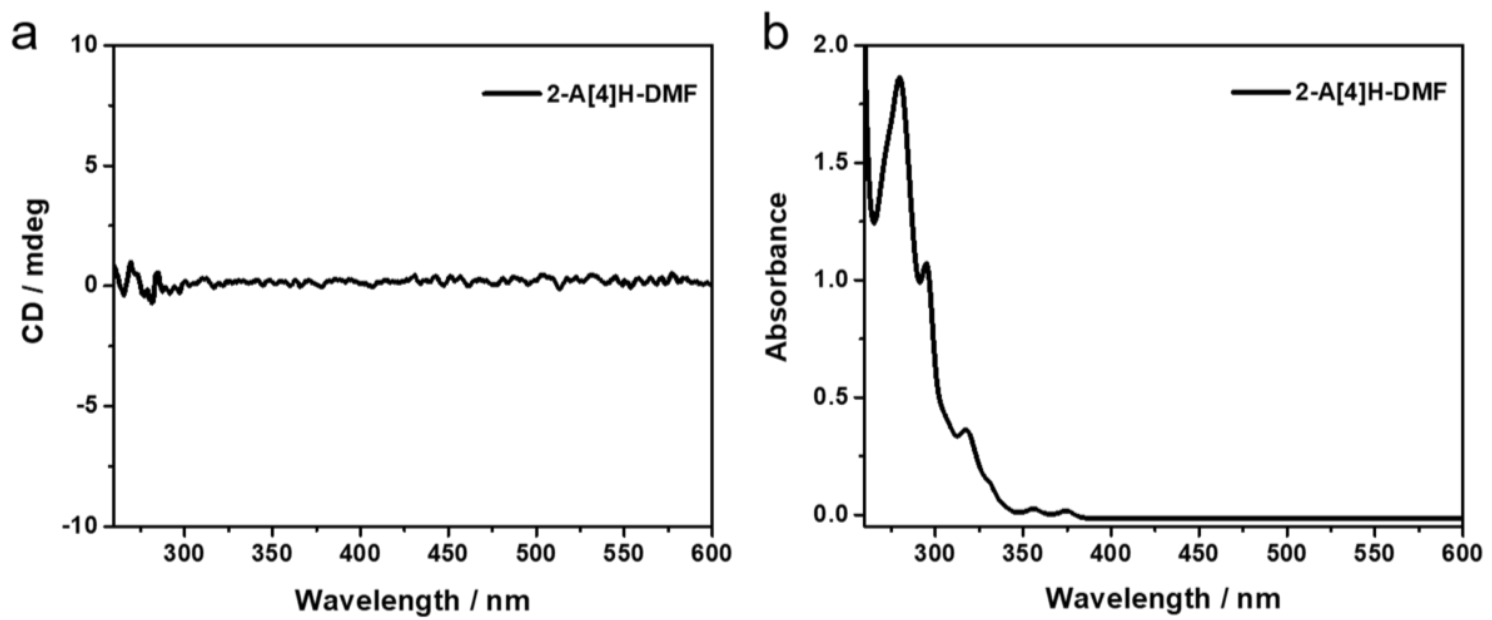

Figure S2. (a) CD and (b) UV-vis spectra of 2-A[4]H in DMF with a concentration of $0.22 \times 10^{-3} \mathrm{~mol} \cdot \mathrm{L}^{-1}$.

\section{Racemization barriers of 2-A[4]H and 4-A[4]H}

Density functional theory (DFT) calculations were carried out using Gaussian 09 program. Geometrical optimization calculations were carried out at the PBE0-D3(BJ)/def2-SVP level without any symmetry assumptions unless otherwise stated. Harmonic vibration frequency calculations were performed at the same level for verifying the resulting geometries as local minima (with all the frequencies real) or saddle points (with only one imaginary frequency). The assignment of the saddle points was performed using intrinsic reaction coordinate (IRC) calculations. 
Table S1. Calculated enthalpies $\Delta H^{\ddagger}$ calc and free energies $\Delta G^{\ddagger}{ }_{\text {calc }}$ (in $\mathrm{kcal} \cdot \mathrm{mol}^{-1}$ at $298.15 \mathrm{~K}$ )

\begin{tabular}{c|c|c}
\hline & $\Delta H^{\ddagger}{ }_{\text {calc }}$ & $\Delta G^{\ddagger}{ }_{\text {calc }}$ \\
\hline $2-\mathrm{A}[4] \mathrm{H}$ & 3.58 & 4.25 \\
$4-\mathrm{A}[4] \mathrm{H}$ & 3.48 & 4.15 \\
\hline
\end{tabular}

\section{Cartesian coordinates of the optimized geometries}

\section{2-A[4]H}
C $0.021488 \quad 1.8127850 .002846$
C $0.009298 \quad 0.398565 \quad 0.002990$
C $1.288147-0.278508-0.049836$
C $2.487419 \quad 0.457126 \quad 0.177982$
C $2.4332391 .873094 \quad 0.333319$
C $1.246850 \quad 2.523917 \quad 0.186051$
C-1.189985 $2.545360-0.176388$
C-2.382068 $1.904578-0.321267$
C-2.463353 $0.488991-0.170825$
C-1.274669-0.271308 0.057403

C -3.721636-0.156259-0.178136

$H-1.135146 \quad 3.636007-0.216368$

C -3.834514-1.500408 0.099380

$\mathrm{H}-3.3019912 .467017-0.498388$

C - $2.681913-2.233348 \quad 0.434351$

$\mathrm{H}-4.612038 \quad 0.443861-0.382506$

C-1.439574 -1.633140 0.416828

$\mathrm{H}-4.813517-1.984817 \quad 0.099082$

C $1.464814-1.633360-0.442906$

$H-2.768445-3.281483 \quad 0.729650$

N 2.618419-2.263658-0.471413

H $-0.577115-2.2140190 .737959$

C $3.723962-1.588134-0.113372$

H $0.606205-2.201130-0.809727$

C $3.713154-0.242560 \quad 0.178681$

H 3.3599992 .4224110 .514138

H $1.205211 \quad 3.615298 \quad 0.229112$

H $4.660797-2.155690-0.107230$

H $4.6431590 .293818 \quad 0.381558$

\section{2-A[4]H (racemization transition state)}
C $0.019584 \quad 1.757825 \quad 0.000140$
C $0.008749 \quad 0.335611 \quad 0.000096$
C $\quad 1.322029-0.318847 \quad 0.000027$
C $2.511716 \quad 0.479625-0.000030$
C $2.444258 \quad 1.899803 \quad 0.000130$
C 1.2339802 .5078590 .000236
C-1.181463 $2.525850 \quad 0.000052$
C-2.396866 $1.926821-0.000189$
C $-2.4897340 .508282-0.000169$
C-1.308526 $-0.309456 \quad 0.000082$

C - $3.781678-0.068528-0.000343$

$H-1.094619 \quad 3.614718 \quad 0.000126$

C -3.963795 -1.430376-0.000193

$H-3.317355 \quad 2.515091-0.000330$

C-2.826556-2.251002 0.000293

$H-4.6414520 .606198-0.000599$

C-1.557791-1.708134 0.000392

C $1.581775-1.7209050 .000018$

N $2.767727-2.291261-0.000110$

$H-4.966437-1.863110-0.000373$

C $3.860399-1.515222-0.000259$

$\mathrm{H}-2.934774-3.338026 \quad 0.000628$

C $3.777117-0.144699-0.000192$

$H-0.749093-2.422434 \quad 0.000871$

H $3.372478 \quad 2.475288 \quad 0.000155$

H $\quad 0.773144-2.440936 \quad 0.000120$

H $4.828587-2.027264-0.000405$

H $1.158936 \quad 3.5978390 .000293$

\section{4-A[4]H}
C $\quad 0.025617 \quad 1.803068 \quad 0.006355$
C $0.0055350 .389262 \quad 0.005079$
C $1.279254-0.298304-0.053102$

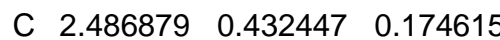

C $2.437388 \quad 1.850474 \quad 0.321159$

C-2.467258 $0.490979-0.173044$

C $1.255355 \quad 2.5091690 .180321$

C-1.282246 $-0.274195 \quad 0.056752$

C-1.183441 $2.540696-0.170865$

C $-3.728386-0.148593-0.184208$

C-2.378593 $1.906630-0.319458$

C-3.849163-1.492451 0.092007 

C - $2.700915-2.229803 \quad 0.431568$
C-1.455763-1.634707 0.417308
C $1.449908-1.649731-0.439299$
C $2.705469-2.217716-0.445579$
C $3.806878-1.426203-0.069636$
N $3.707926-0.1454290 .200410$

H 3.3829652 .3677490 .490550

H $1.220216 \quad 3.600870 \quad 0.219454$

$H-1.1230593 .631095-0.209345$

$H-3.2952642 .474289-0.496989$

$H-4.615373 \quad 0.456146-0.390050$

$H-4.830467-1.9721190 .088869$
$H-2.793688-3.276626 \quad 0.730060$

$H-0.598202-2.217163 \quad 0.748696$

H $0.596489-2.232885-0.781786$

H $2.850417-3.254687-0.755226$

H $\quad 4.811374-1.864807-0.026943$

\section{4-A[4]H (racemization transition state)}
C $0.023764 \quad 1.751052-0.000032$
C $0.006349 \quad 0.329236 \quad 0.000176$
C $1.313525-0.334161-0.000022$

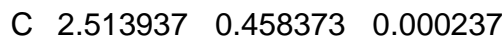
C $2.447596 \quad 1.879474 \quad 0.000034$
C $1.2415332 .495309-0.000264$
C-1.175510 $2.522034 \quad 0.000009$
C -2.393035 $1.927300-0.000116$
C-2.491600 $0.508863-0.000138$
C-1.312962 -0.312345 0.000226

C - $3.785504-0.063237-0.000187$

C -3.973465-1.424434-0.000097

C $-2.839215-2.248758 \quad 0.000318$

C-1.568348-1.709993 0.000616

C $1.564328-1.730801-0.000695$

C $2.844608-2.240306-0.000607$

C $3.929442-1.351943 \quad 0.000234$

N $3.765706-0.051978 \quad 0.000508$

H 3.3942312 .4210620 .000165

H $1.172432 \quad 3.585657-0.000374$
$H-1.085024 \quad 3.610600 \quad 0.000064$

$H-3.3114062 .518933-0.000322$

$\mathrm{H}-4.642648 \quad 0.614877-0.000342$

$H-4.977698-1.853381-0.000193$

$\mathrm{H}-2.951721-3.335434 \quad 0.000503$

$\mathrm{H}-0.764656-2.429451 \quad 0.001317$

H $0.765260-2.456868-0.001895$

H $3.008226-3.319851-0.001158$

H $4.960406-1.726270 \quad 0.000524$ a

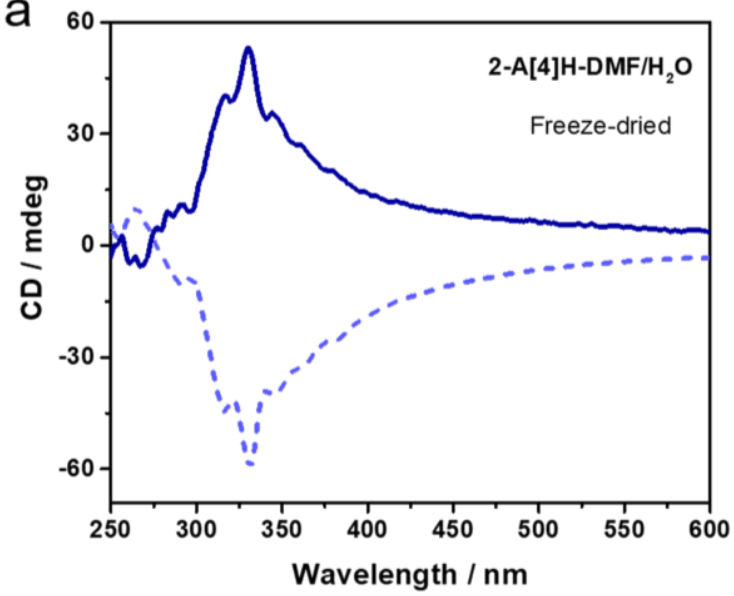

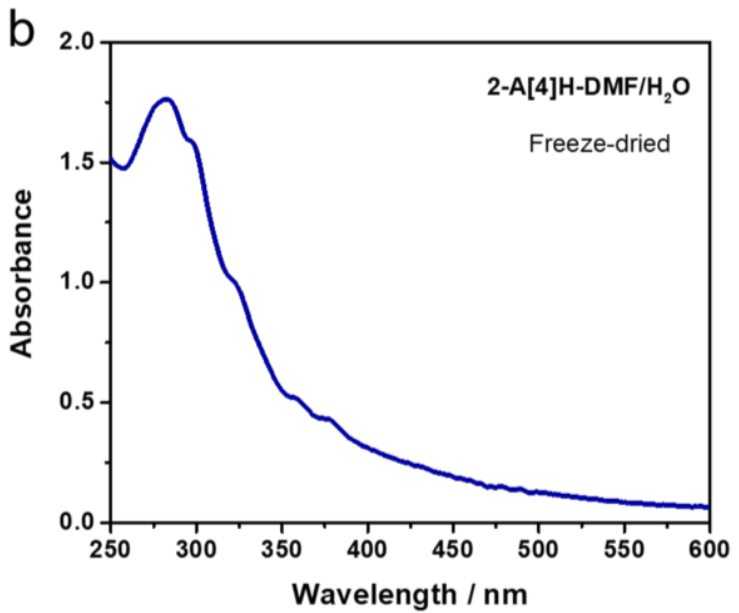

Figure S3. (a) CD and (b) UV-vis spectra of freeze-dried 2-A[4]H aggregates formed in $\mathrm{DMF} / \mathrm{H}_{2} \mathrm{O}(1 / 99$, $\mathrm{v} / \mathrm{v})$ with a concentration of $1.3 \times 10^{-3} \mathrm{~mol} \cdot \mathrm{L}^{-1}$. 

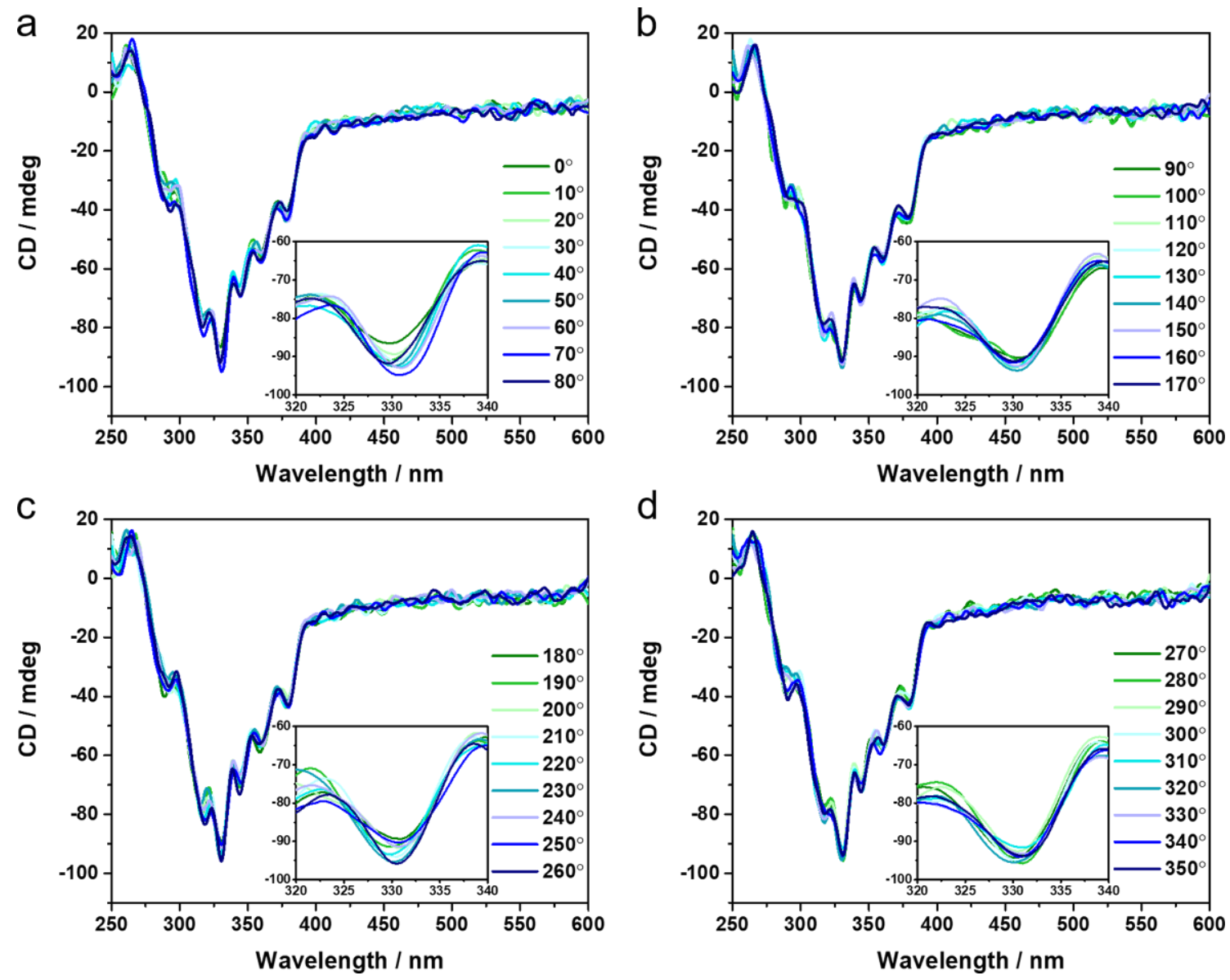

Figure S4. $\mathrm{CD}$ spectra of a plate formed by 2-A[4]H aggregates and $\mathrm{KBr}$ at different rotating angles in the range of (a) $0^{\circ} \sim 80^{\circ}$, (b) $90^{\circ} \sim 170^{\circ}$, (c) $180^{\circ} \sim 260^{\circ}$, (d) $270^{\circ} \sim 350^{\circ}$, with an interval of $10^{\circ}$. The 36 individual CD spectra showed slightly different CD amplitude, indicating a weak LD effect.

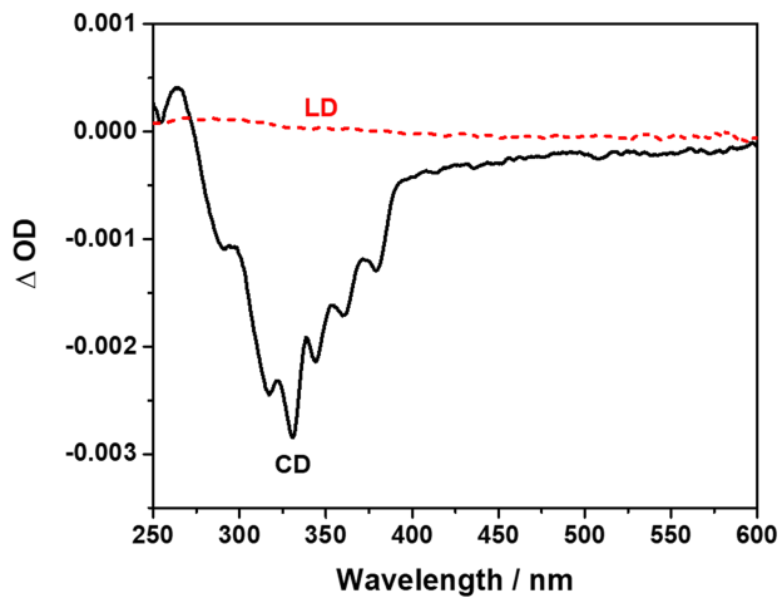

Figure S5. Mean spectrum of all $36 \mathrm{CD}$ spectra (in $\triangle \mathrm{OD}$, solid black line) for comparison with mean spectrum of all 36 LD spectra (dash red line). To investigate the contribution of LD on the CD signals, the film of $2-\mathrm{A}[4] \mathrm{H}$ aggregate was placed in different angles and average $\mathrm{CD}$ and $\mathrm{LD}$ signals were taken according to reported procedures in literature. ${ }^{\mathrm{S2}}$ Compared to the mean spectrum of all $36 \mathrm{CD}$ spectra, the mean spectrum of all 36 LD spectra was in much low intensity and thus the contribution was negligible. 

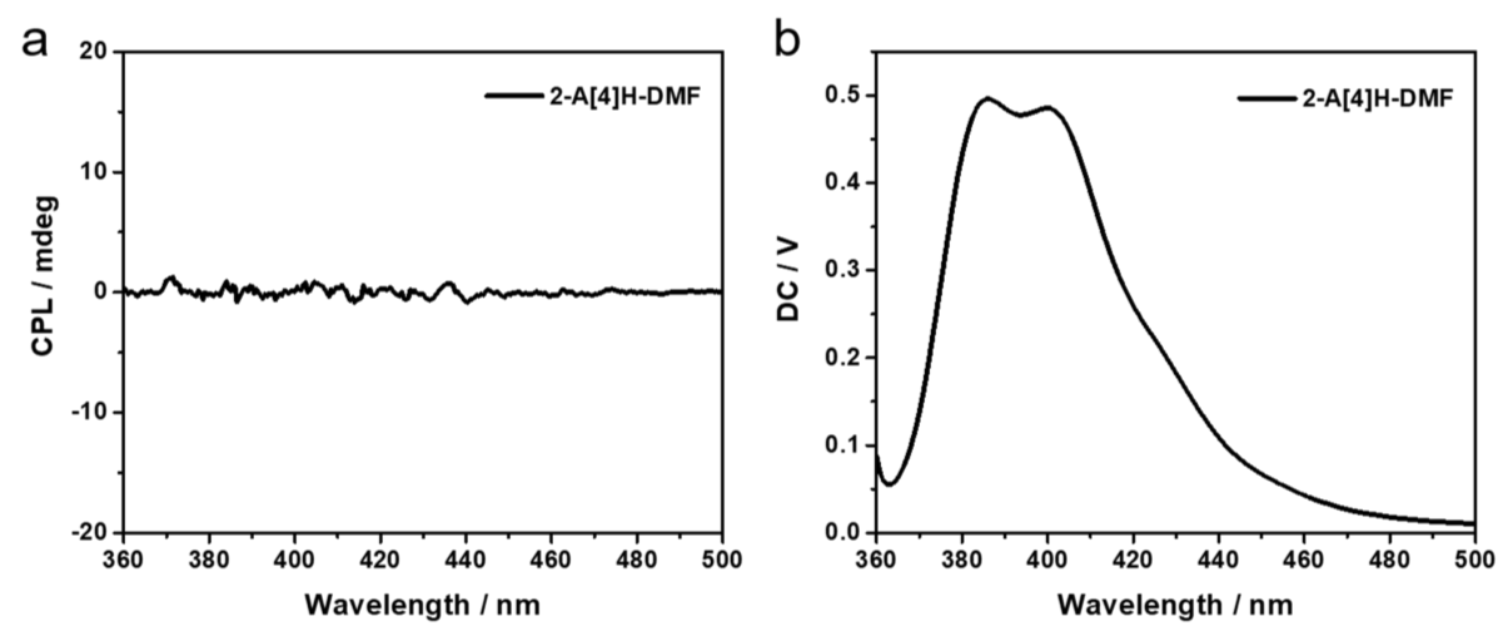

Figure S6. (a) $\mathrm{CPL}$ and (b) fluorescence spectra of $2-\mathrm{A}[4] \mathrm{H}$ in DMF with a concentration of $0.22 \times 10^{-3}$ $\mathrm{mol} \cdot \mathrm{L}^{-1}$.
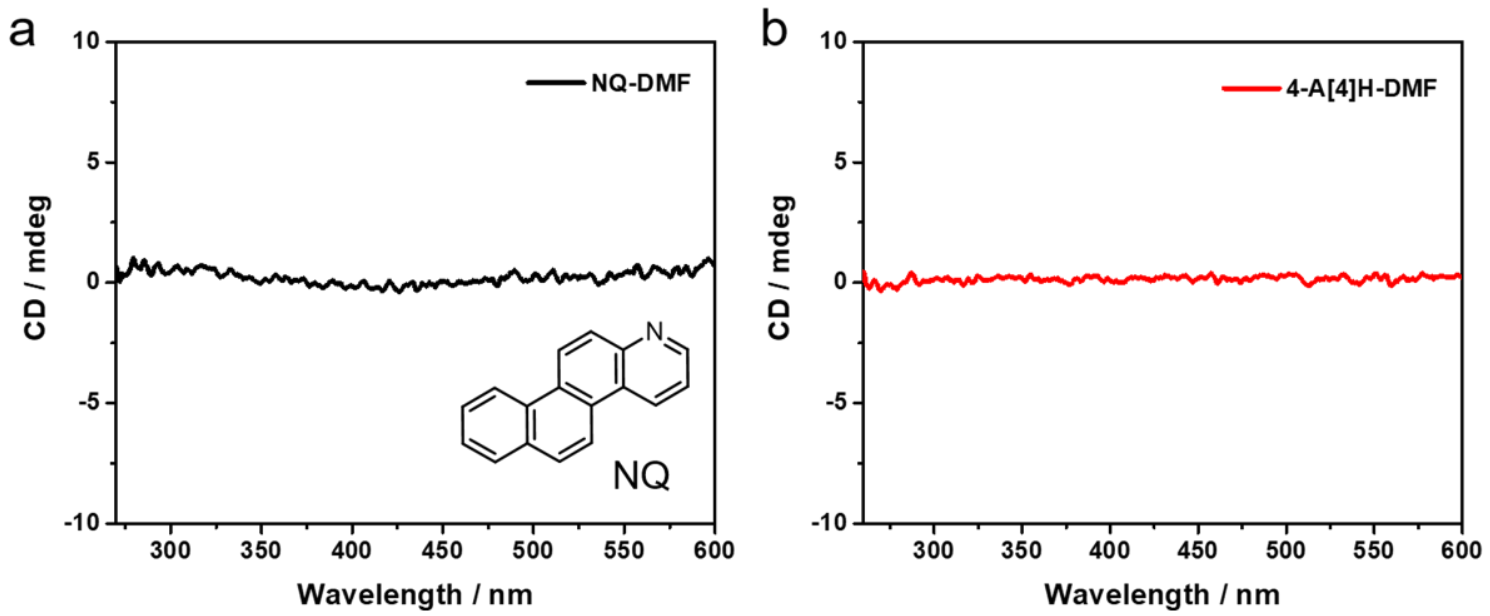

Figure S7. CD spectra of (a) NQ and (b) 4-A[4] $\mathrm{H}$ in DMF with a concentration of $0.22 \times 10^{-3} \mathrm{~mol} \cdot \mathrm{L}^{-1}$.
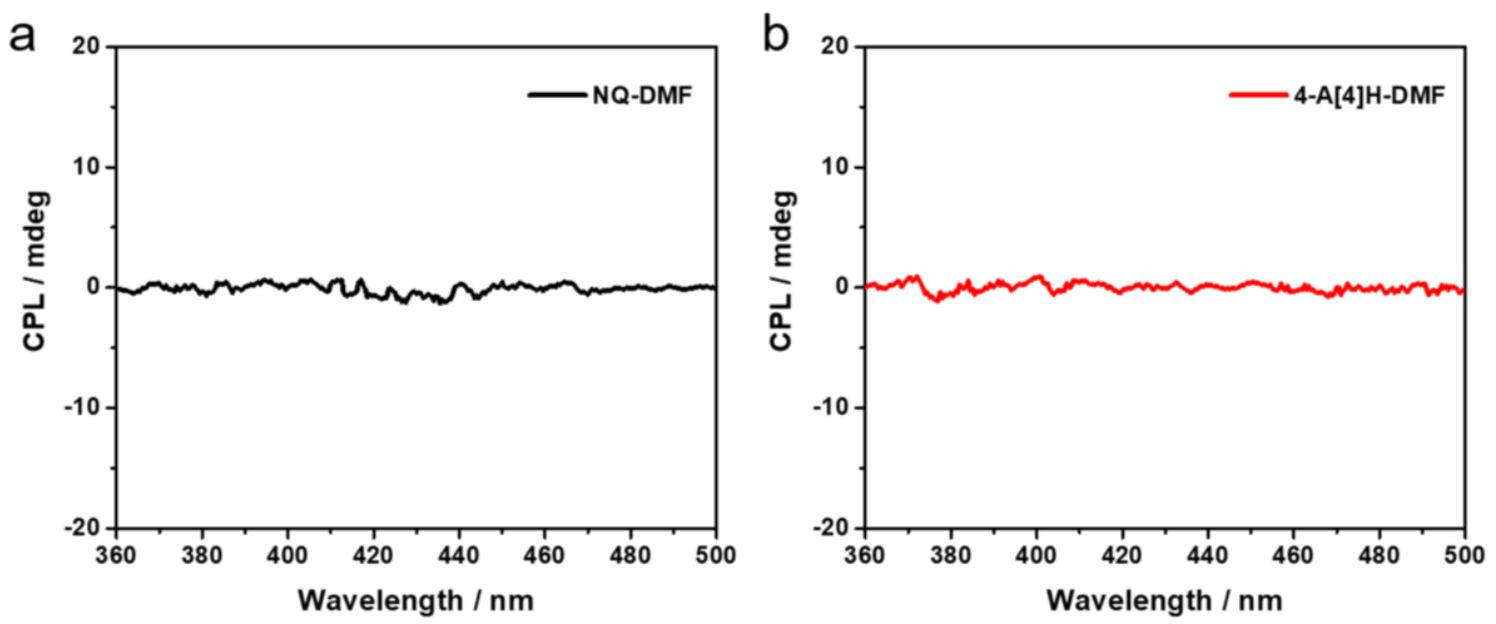

Figure S8. CPL spectra of (a) NQ and (b) 4-A[4]H in DMF with a concentration of $0.22 \times 10^{-3} \mathrm{~mol}^{-\mathrm{L}^{-1}}$. 
a

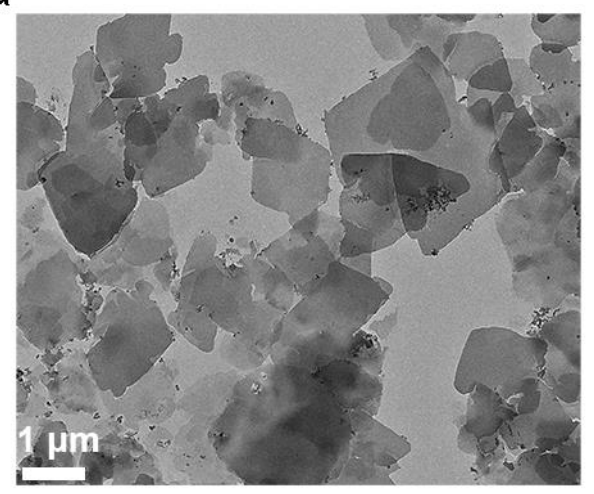

b

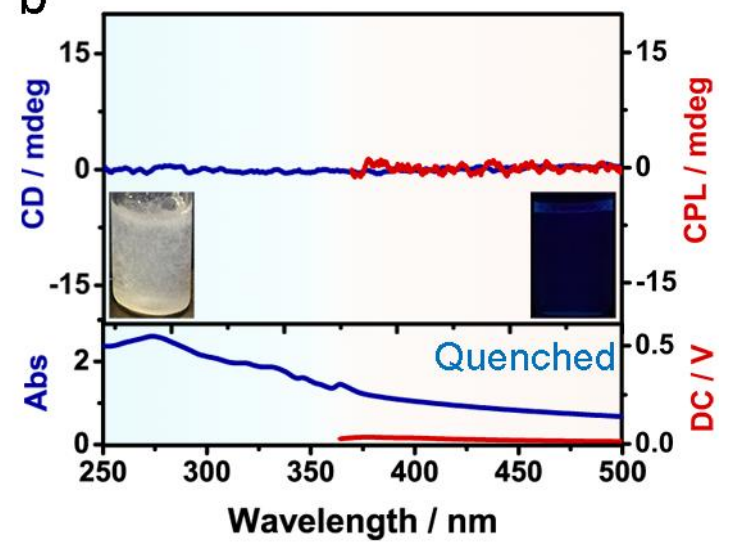

Figure S9. (a) TEM image of $N Q$ aggregates formed in $D M F / H_{2} \mathrm{O}(1 / 99, v / v)$. (b) UV-vis, $C D$, fluorescence, and CPL spectra of NQ aggregates. The insets are the photographs of NQ aggregates before and after UV irradiation.
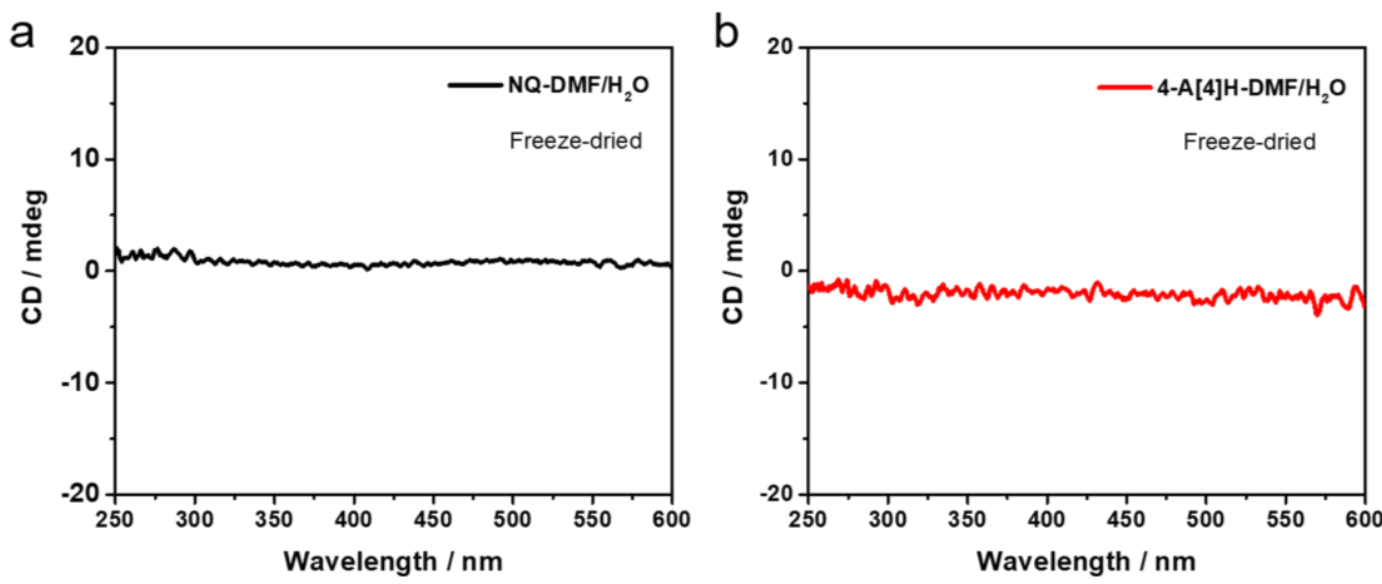

Figure S10. CD spectra of freeze-dried (a) NQ aggregates and (b) $4-A[4] \mathrm{H}$ crystals formed in $\mathrm{DMF} / \mathrm{H}_{2} \mathrm{O}$ $(1 / 99, \mathrm{v} / \mathrm{v})$ with a concentration of $1.3 \times 10^{-3} \mathrm{~mol} \cdot \mathrm{L}^{-1}$.

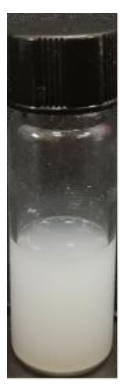

$0 \mathrm{~h}$

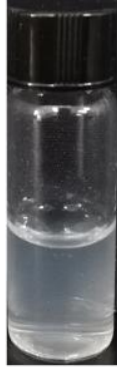

$6 \mathrm{~h}$

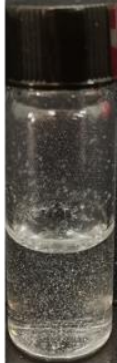

$48 \mathrm{~h}$

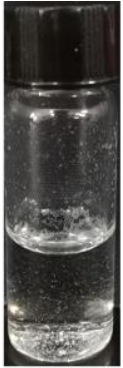

$>48 \mathrm{~h}$

Figure S11. Photographs of 2-A[4] $\mathrm{H}$ aggregates formed at different time periods. After the addition of water into a DMF solution 2- $\mathrm{A}[4] \mathrm{H}$ initially formed a white turbid dispersion and gradually transformed into a suspension containing visible small-size aggregates. 


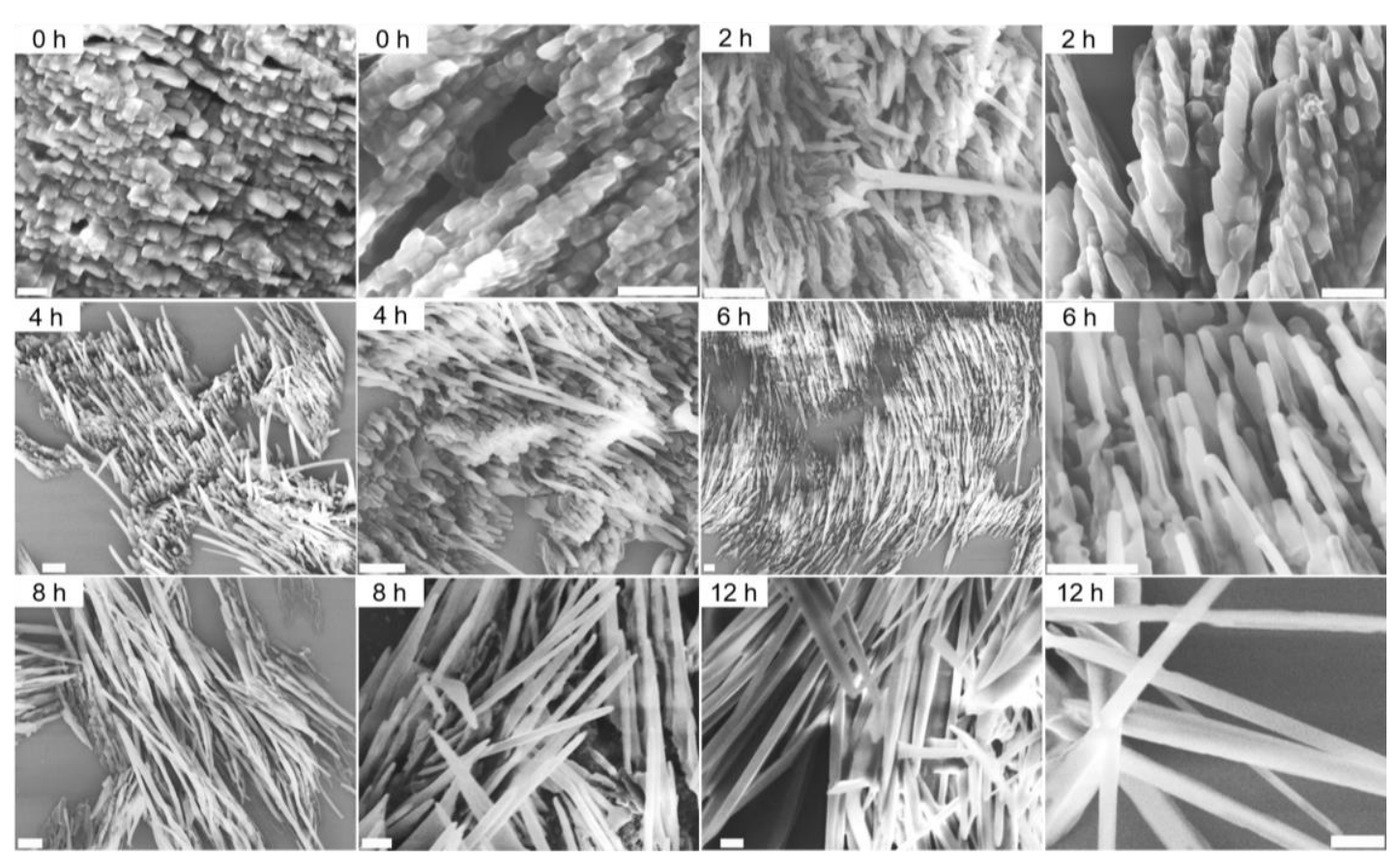

Figure S12. Additional SEM images of various aggregates formed by $2-\mathrm{A}[4] \mathrm{H}$ in $\mathrm{DMF} / \mathrm{H}_{2} \mathrm{O}(1 / 99, \mathrm{v} / \mathrm{v})$ at different time periods. Scale bar $=1 \mu \mathrm{m}$. The concentration of $2-\mathrm{A}[4] \mathrm{H}$ is $1.3 \times 10^{-3} \mathrm{~mol} \cdot \mathrm{L}^{-1}$.
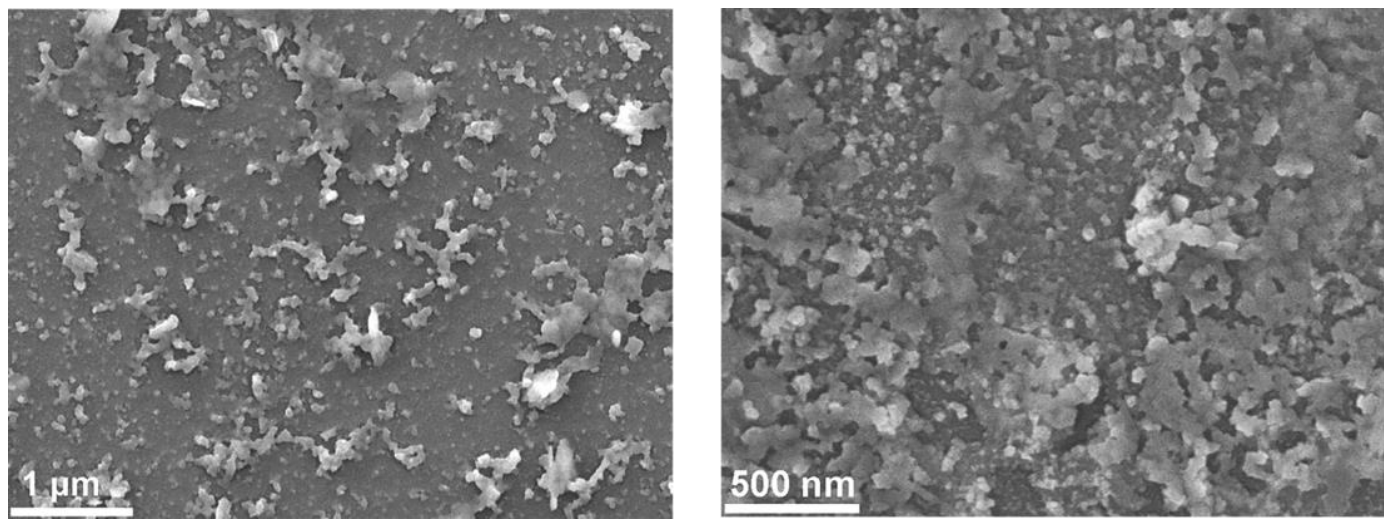

Figure S13. SEM images of dried 2-A[4] H aggregates obtained in $\mathrm{DMF} / \mathrm{H}_{2} \mathrm{O}(1 / 99, \mathrm{v} / \mathrm{v})$ around $70{ }^{\circ} \mathrm{C}$ with a concentration of $1.3 \times 10^{-3} \mathrm{~mol} \cdot \mathrm{L}^{-1}$. 
Table S2. Crystal data and structure refinement for a single crystal of 2-A[4]H formed in acetone/n-pentane

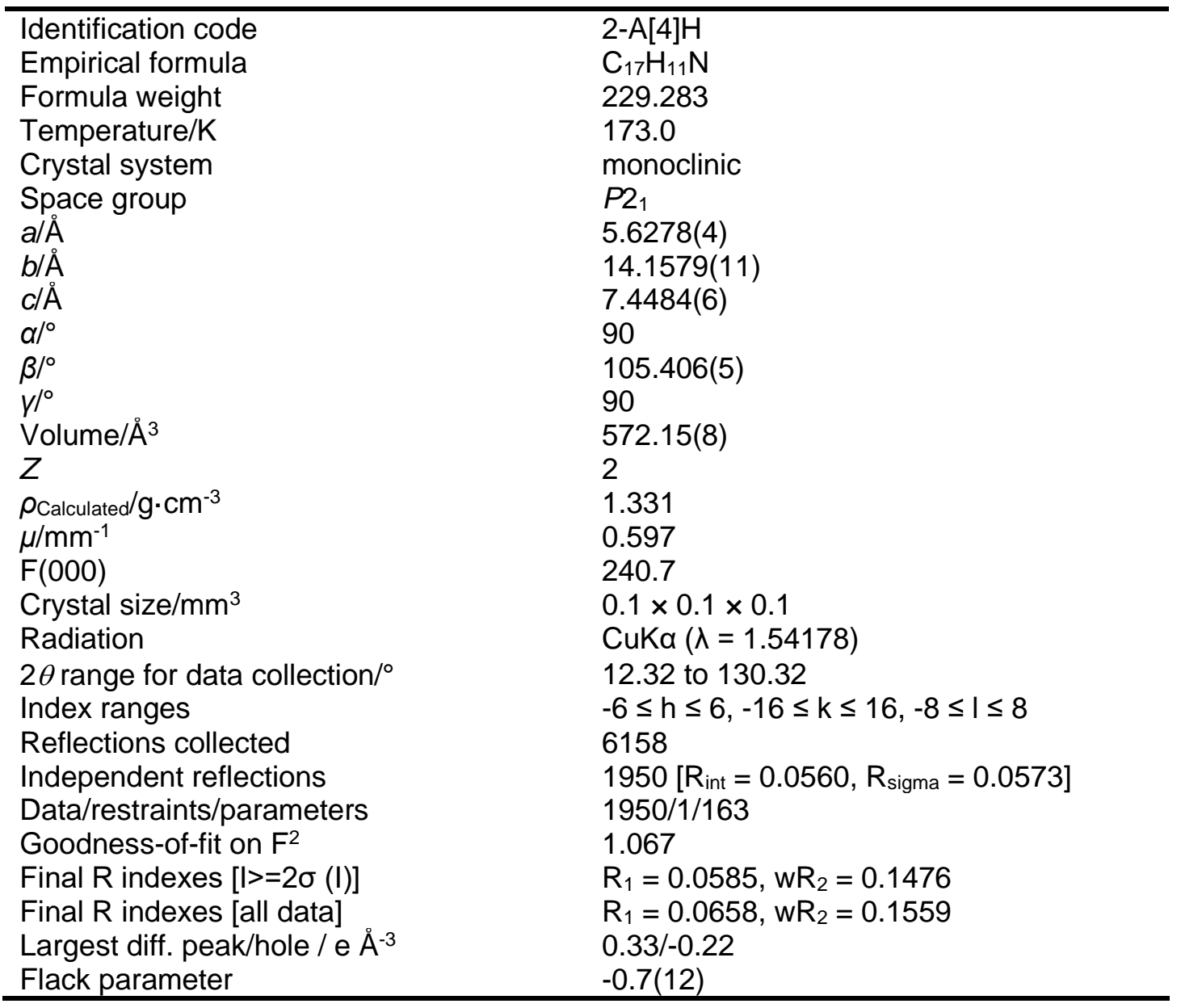

It should be noted that due to the limited quality of the obtained crystals, the high angle data was dominated by noise. As a concequnce, one "Alert level A" occured after the checkCIF procedure.
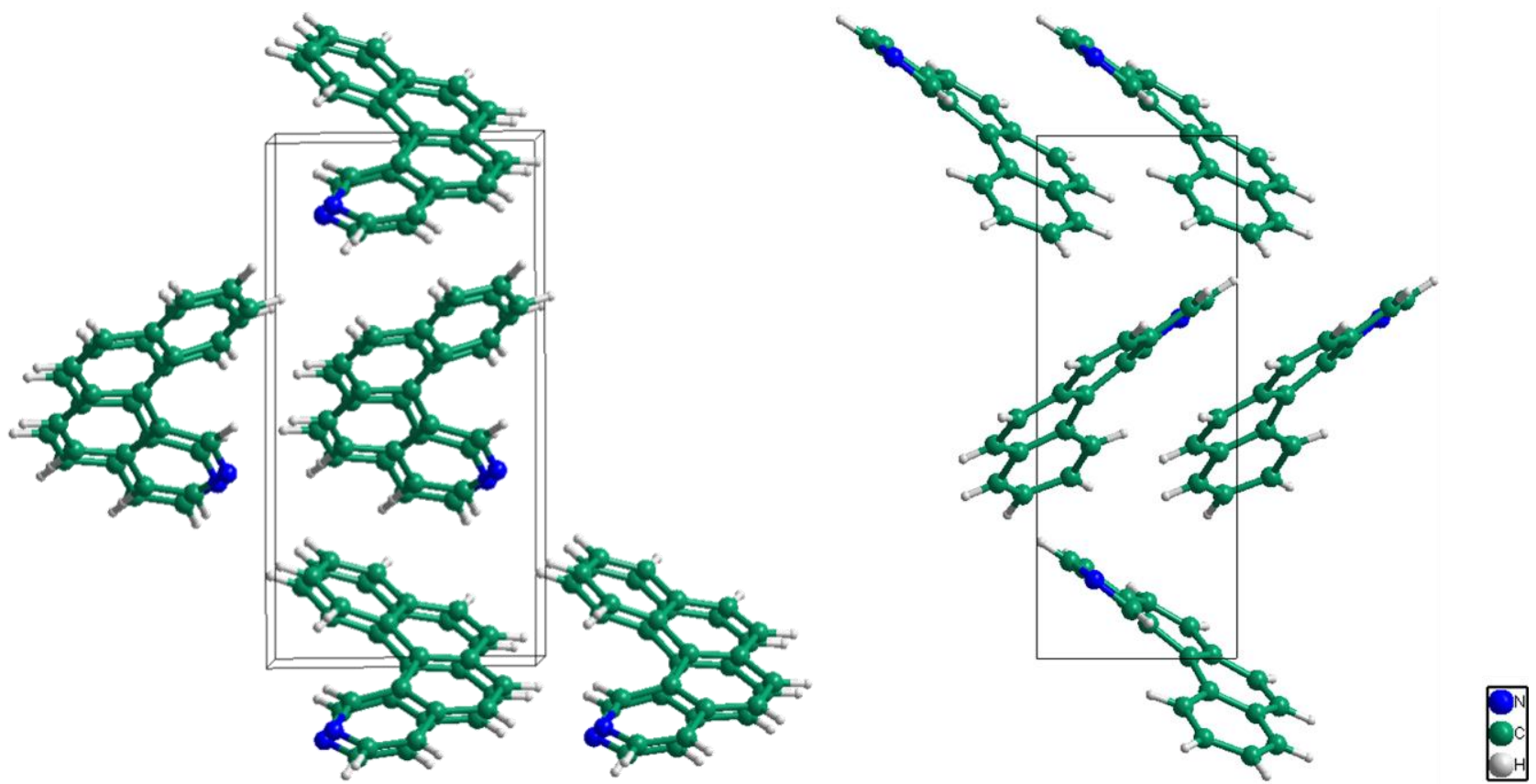

Figure S14. Single-crystal structure of $2-\mathrm{A}[4] \mathrm{H}$ obtained from an acetone/ $n$-pentane mixture by gaseous phase diffusion. 


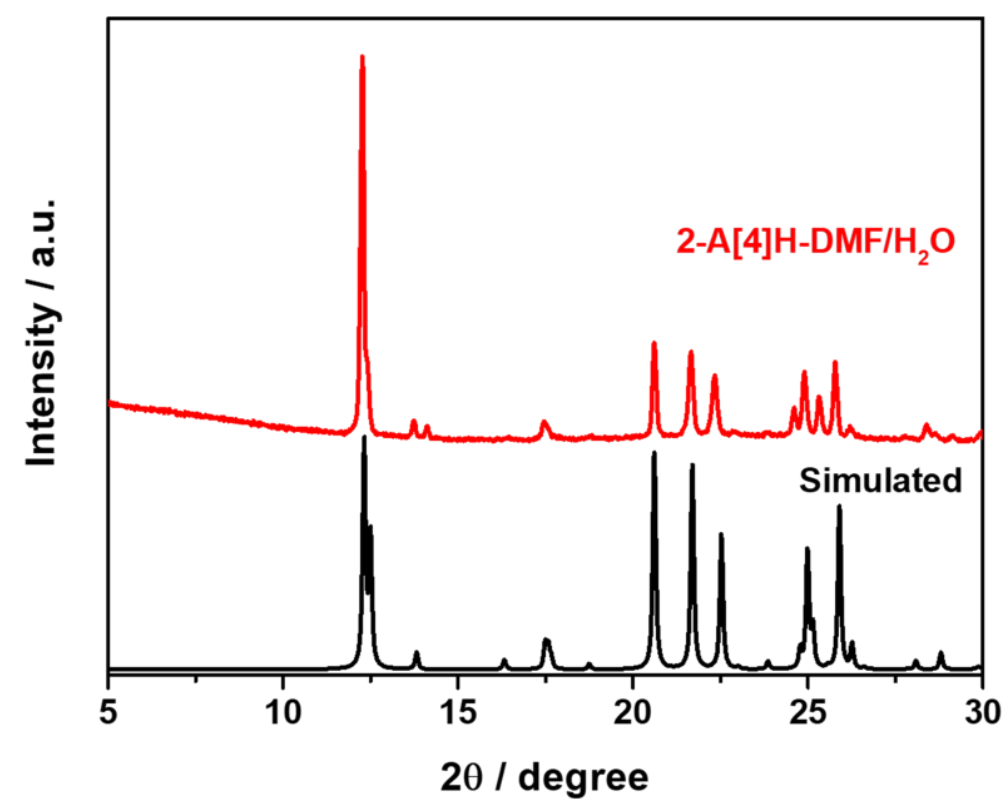

Figure S15. Comparison between simulated powder XRD pattern of a $2-\mathrm{A}[4] \mathrm{H}$ crystal formed in a acetone/n-pentane mixture and measured powder $\mathrm{XRD}$ pattern of $2-\mathrm{A}[4] \mathrm{H}$ aggregates prepared in $\mathrm{DMF} / \mathrm{H}_{2} \mathrm{O}(1 / 99, \mathrm{v} / \mathrm{v})$.

Table S3. Crystal data and structure refinement for a single crystal of 4-A[4] $\mathbf{H}$ formed in $\mathrm{DMF} / \mathrm{H}_{2} \mathrm{O}$

\begin{tabular}{|c|c|}
\hline Identification code & $4-\mathrm{A}[4] \mathrm{H}$ \\
\hline Empirical formula & $\mathrm{C}_{17} \mathrm{H}_{11} \mathrm{~N}$ \\
\hline Formula weight & 229.283 \\
\hline Temperature/K & 191.65 \\
\hline Crystal system & orthorhombic \\
\hline Space group & Pna2 1 \\
\hline$a / \AA$ & $9.7299(19)$ \\
\hline$b / \AA$ & $22.520(5)$ \\
\hline$c / \AA$ & $5.2706(11)$ \\
\hline$\alpha /^{\circ}$ & 90 \\
\hline$\beta /{ }^{\circ}$ & 90 \\
\hline$y^{\circ}$ & 90 \\
\hline Volume/Ä3 & $1154.9(4)$ \\
\hline$Z$ & 4 \\
\hline$\rho_{\text {Calculated }} / \mathrm{g} \cdot \mathrm{cm}^{-3}$ & 1.319 \\
\hline$\mu / \mathrm{mm}^{-1}$ & 0.592 \\
\hline$F(000)$ & 481.4 \\
\hline Crystal size $/ \mathrm{mm}^{3}$ & $0.12 \times 0.1 \times 0.08$ \\
\hline Radiation & CuKa $(\lambda=1.54178)$ \\
\hline $2 \theta$ range for data collection $/{ }^{\circ}$ & 7.86 to 127.3 \\
\hline Index ranges & $-11 \leq \mathrm{h} \leq 9,-25 \leq \mathrm{k} \leq 26,-5 \leq \mathrm{I} \leq 5$ \\
\hline Reflections collected & 5796 \\
\hline Independent reflections & $1747\left[R_{\text {int }}=0.0605, R_{\text {sigma }}=0.0614\right]$ \\
\hline Data/restraints/parameters & $1747 / 2 / 163$ \\
\hline Goodness-of-fit on $\mathrm{F}^{2}$ & 1.059 \\
\hline Final $R$ indexes $[I>=2 \sigma(I)]$ & $\mathrm{R}_{1}=0.0600, \mathrm{wR}_{2}=0.1774$ \\
\hline Final $R$ indexes [all data] & $R_{1}=0.0663, w R_{2}=0.1890$ \\
\hline Largest diff. peak/hole/e. $\AA^{-3}$ & $0.19 /-0.19$ \\
\hline Flack parameter & $-2.7(17)$ \\
\hline
\end{tabular}



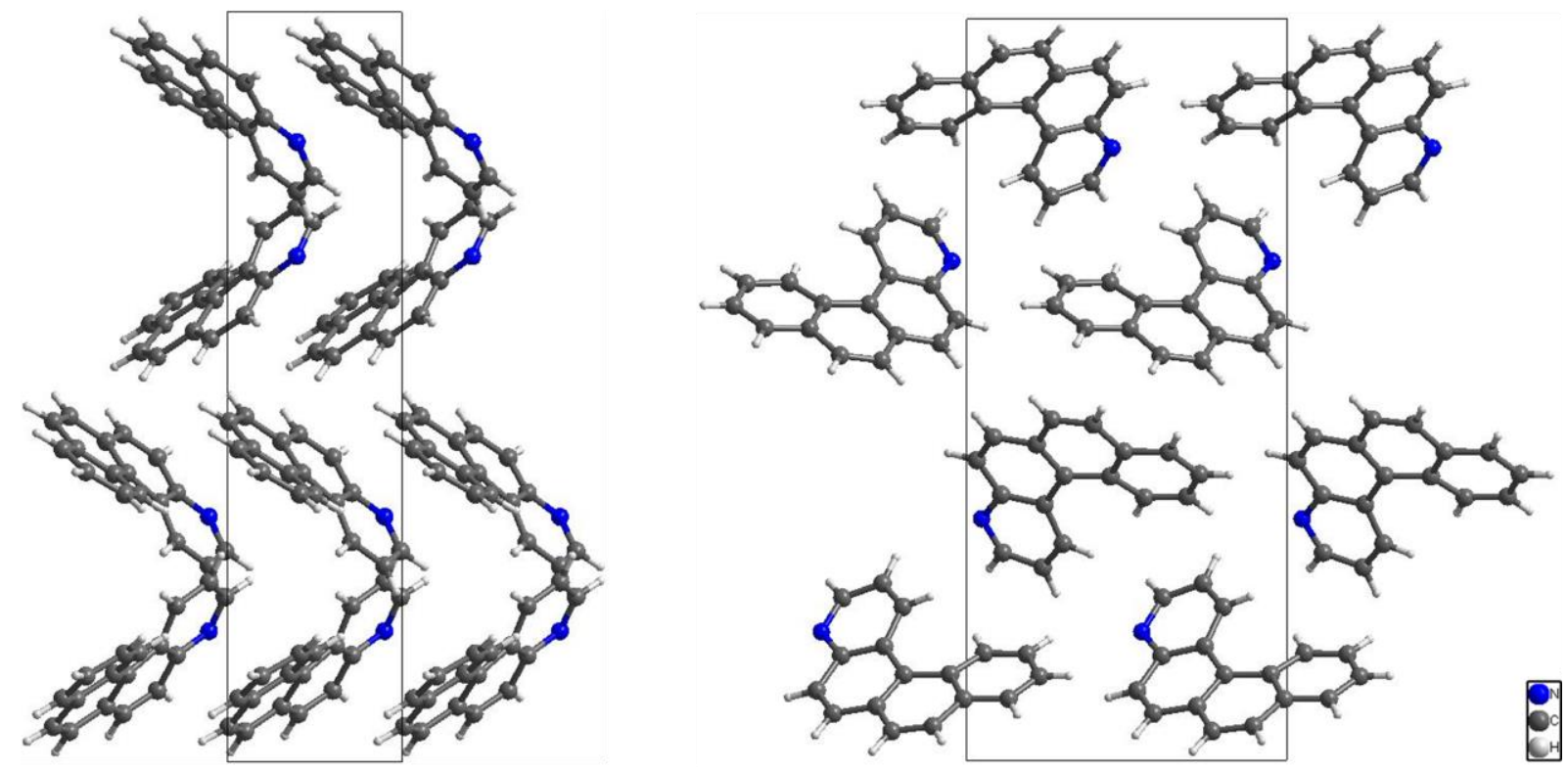

Figure S16. Packing structure of 4-A[4] $\mathrm{H}$ in a crystal obtained from a $\mathrm{DMF} / \mathrm{H}_{2} \mathrm{O}(1 / 99, \mathrm{v} / \mathrm{v})$ mixture.

Table S4. Crystal data and structure refinement for a single crystal of 4-A[4]H formed in dichloromethane/npentane

\begin{tabular}{|c|c|}
\hline Identification code & $4-\mathrm{A}[4] \mathrm{H}$ \\
\hline Empirical formula & $\mathrm{C}_{17} \mathrm{H}_{11} \mathrm{~N}$ \\
\hline Formula weight & 229.27 \\
\hline Temperature/K & 293(2) \\
\hline Crystal system & orthorhombic \\
\hline Space group & $P_{n a 2}$ \\
\hline$a / \AA$ & $9.6945(17)$ \\
\hline$b / \AA$ & $22.594(7)$ \\
\hline$c / \AA$ & $5.1731(15)$ \\
\hline$\alpha /{ }^{\circ}$ & 90 \\
\hline$\beta /^{\circ}$ & 90 \\
\hline$y^{\circ}$ & 90 \\
\hline Volume/Å3 & $1133.1(5)$ \\
\hline$Z$ & 4 \\
\hline$\rho_{\text {Calculated }} / \mathrm{g} \cdot \mathrm{cm}^{-3}$ & 1.344 \\
\hline$\mu / \mathrm{mm}^{-1}$ & 0.603 \\
\hline$F(000)$ & 480.0 \\
\hline Crystal size $/ \mathrm{mm}^{3}$ & $0.3 \times 0.2 \times 0.2$ \\
\hline Radiation & $\operatorname{CuKa}(\lambda=1.54178)$ \\
\hline $2 \theta$ range for data collection ${ }^{\circ}$ & 7.826 to 157.57 \\
\hline Index ranges & $-12 \leq \mathrm{h} \leq 12,-28 \leq \mathrm{k} \leq 28,-5 \leq \mathrm{I} \leq 6$ \\
\hline Reflections collected & 17664 \\
\hline Independent reflections & $2245\left[R_{\text {int }}=0.0557, R_{\text {sigma }}=0.0321\right]$ \\
\hline Data/restraints/parameters & $2245 / 1 / 163$ \\
\hline Goodness-of-fit on $\mathrm{F}^{2}$ & 0.896 \\
\hline Final $R$ indexes $[\mid>=2 \sigma(I)]$ & $R_{1}=0.0360, w R_{2}=0.1031$ \\
\hline Final $R$ indexes [all data] & $\mathrm{R}_{1}=0.0405, \mathrm{wR}_{2}=0.1088$ \\
\hline Largest diff. peak/hole/e. $\AA^{-3}$ & $0.15 /-0.19$ \\
\hline Flack parameter & $0.0(5)$ \\
\hline
\end{tabular}



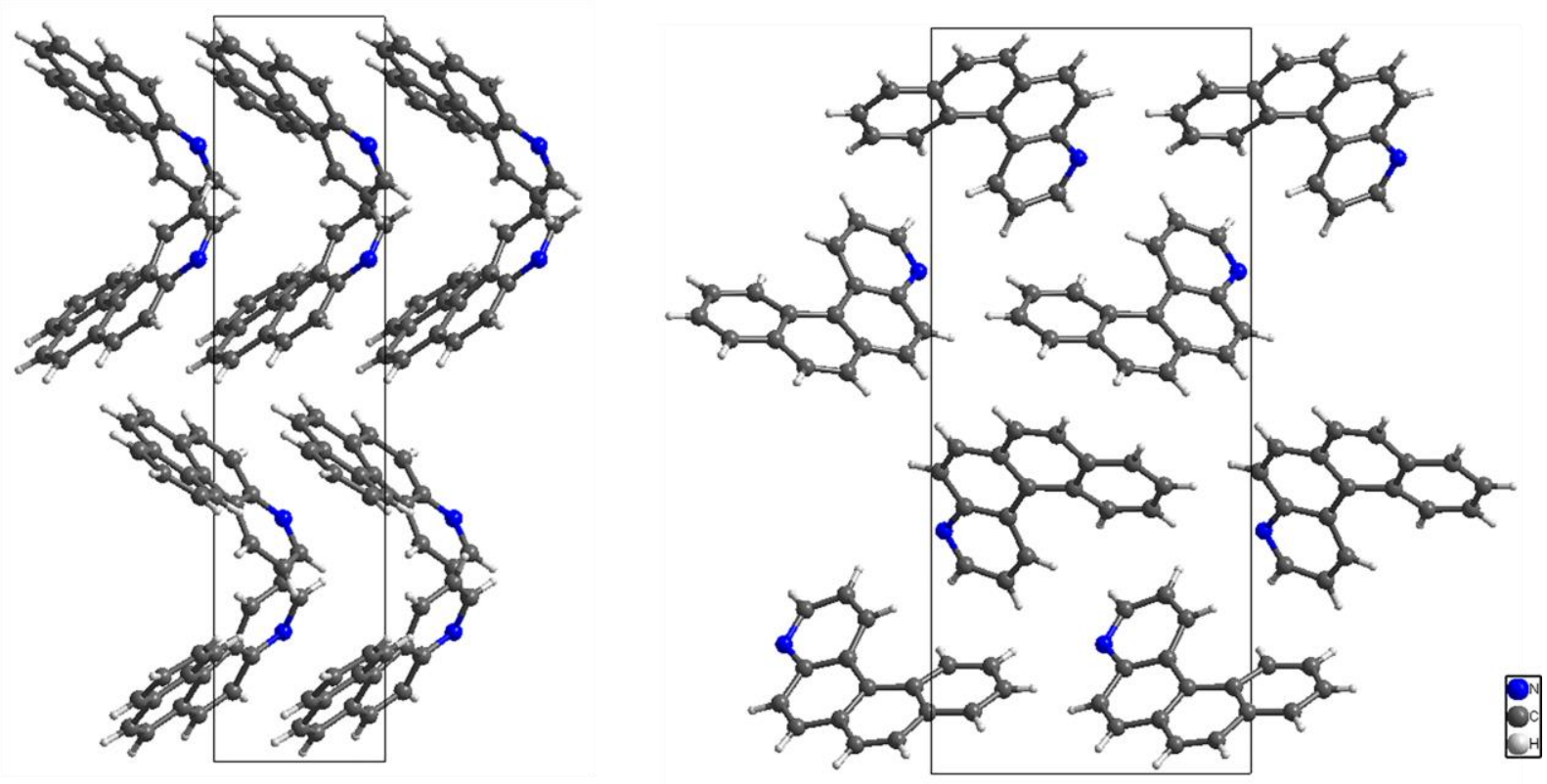

Figure S17. Packing structure of 4-A[4] $\mathrm{H}$ in a crystal obtained from a dichloromethane/n-pentane mixture by gaseous phase diffusion.

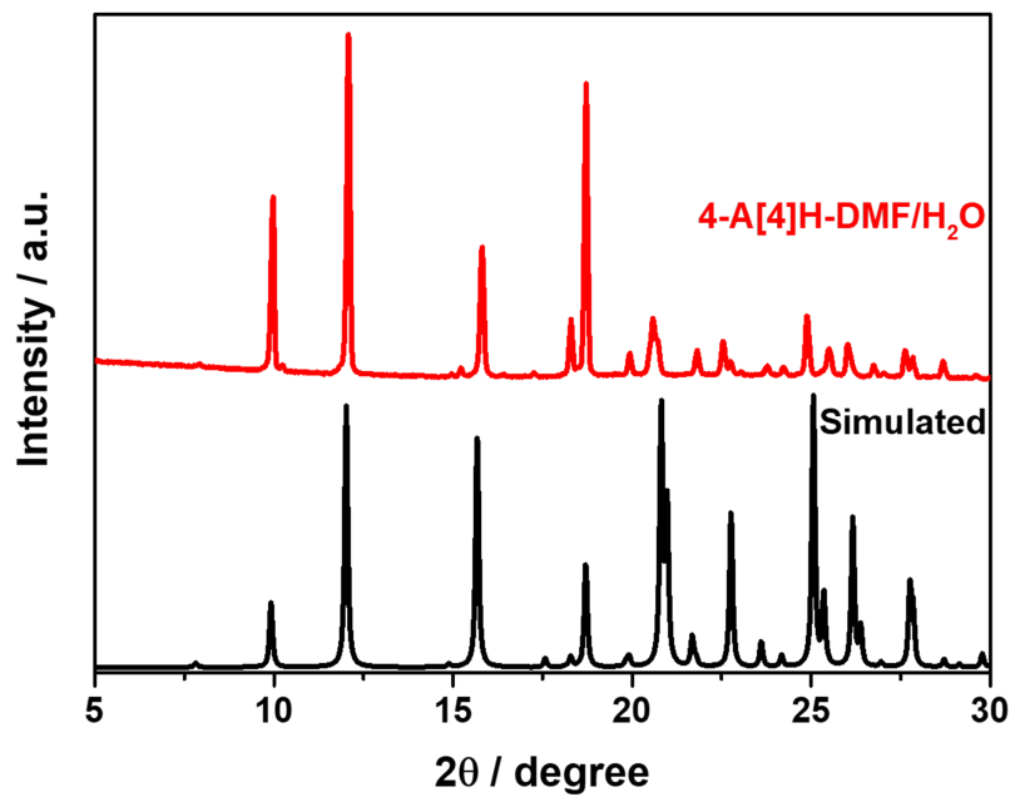

Figure S18. Comparison between simulated powder XRD pattern of a 4-A[4]H crystal formed in a dichloromethane/n-pentane mixture and measured powder XRD pattern of 4-A[4]H crystals obtained in $\mathrm{DMF} / \mathrm{H}_{2} \mathrm{O}(1 / 99, \mathrm{v} / \mathrm{v})$. 


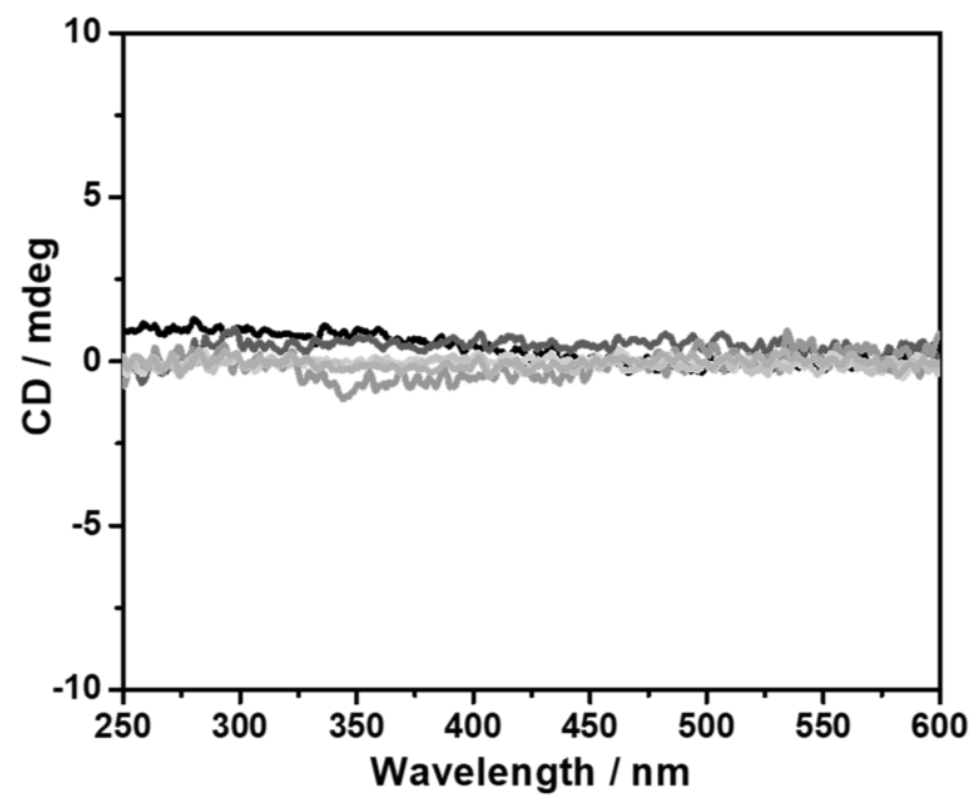

Figure S19. CD spectra of six parallel 2-A[4]H aggregate samples formed in $D M F / \mathrm{H}_{2} \mathrm{O}(1 / 99, \mathrm{v} / \mathrm{v})$ with a concentration of $1.3 \times 10^{-3} \mathrm{~mol} \cdot \mathrm{L}^{-1}$ which were heated to $100^{\circ} \mathrm{C}$ in the initial stage.

\section{References}

S1. Hewlins, M. J. E.; Salter, R. The photochemical cyclodehydrogenation route to polycyclic azaarenes. Synthesis 2007, 14, 2164-2174.

S2. (a) Tsuda, A.; Alam, M. A.; Harada, T.; Yamaguchi, T.; Ishii, N.; Aida, T. Spectroscopic visualization of vortex flows using dye-containing nanofibers. Angew. Chem., Int. Ed. 2007, 46, 8198-8202; (b) Shen, Z.; Jiang, Y.; Wang, T.; Liu, M. Symmetry breaking in the supramolecular gels of an achiral gelator exclusively driven by ח-ா stacking. J. Am. Chem. Soc. 2015, 137, 16109-16115; (c) Spitz, C.; Dähne, S.; Ouart, A.; Abraham, H. W. Proof of chirality of J-aggregates spontaneously and enantioselectively generated from achiral dyes. J. Phys. Chem. B 2000, 104, 8664-8669. 